ARTICLE

Received 6 Jan 2014 | Accepted 5 Jun 2014 | Published 14 Jul 2014

\title{
Lysosome sorting of $\beta$-glucocerebrosidase by LIMP-2 is targeted by the mannose 6-phosphate receptor
}

Yuguang Zhao ${ }^{1, \star}$, Jingshan Ren ${ }^{1, \star}$, Sergi Padilla-Parra ${ }^{1}$, Elizabeth E. Fry ${ }^{1} \&$ David I. Stuart ${ }^{1}$

The integral membrane protein LIMP-2 has been a paradigm for mannose 6-phosphate receptor (MPR) independent lysosomal targeting, binding to $\beta$-glucocerebrosidase ( $\beta$-GCase) and directing it to the lysosome, before dissociating in the late-endosomal/lysosomal compartments. Here we report structural results illuminating how LIMP-2 binds and releases $\beta$-GCase according to changes in $\mathrm{pH}$, via a histidine trigger, and suggesting that LIMP-2 localizes the ceramide portion of the substrate adjacent to the $\beta$-GCase catalytic site. Remarkably, we find that LIMP-2 bears P-Man, $\mathrm{GICNAc}_{2}$ covalently attached to residue N325, and that it binds MPR, via mannose 6-phosphate, with a similar affinity to that observed between LIMP-2 and $\beta$-GCase. The binding sites for $\beta$-GCase and the MPR are functionally separate, so that a stable ternary complex can be formed. By fluorescence lifetime imaging microscopy, we also demonstrate that LIMP-2 interacts with MPR in living cells. These results revise the accepted view of LIMP-2- $\beta$-GCase lysosomal targeting.

\footnotetext{
${ }^{1}$ Division of Structural Biology, University of Oxford, The Henry Wellcome Building for Genomic Medicine, Headington, Oxford OX3 7BN, UK. * These authors contributed equally to this work. Correspondence and requests for materials should be addressed to D.I.S. (email: dave@strubi.ox.ac.uk).
} 
ysosomal integral membrane protein 2 (LIMP-2) is encoded by gene $S C A R B 2$, and is also known as scavenger receptor class B member 2 (SCARB2; refs 1,2). It has $\mathrm{N}$ - and C-terminal transmembrane helices (being a type III membrane protein) and a heavily glycosylated 400 residue luminal domain (bearing nine potential N-linked glycosylation sites) ${ }^{3}$. LIMP-2 has a major role in lysosomal and endosomal membrane organization, and LIMP-2 mutations cause several neurodegenerative and renal diseases, such as myoclonic epilepsy and nephrotic syndrome ${ }^{4,5}$. LIMP-2 is a member of the CD36 superfamily of scavenger receptors ${ }^{6,7}$, and has been identified as a receptor for Enterovirus 71 (EV71) and Coxsackievirus A16, which cause major epidemics of hand, foot-and-mouth disease in young children ${ }^{8}$. Structure determination of the lysosomal domain of LIMP-2 at $\mathrm{pH} 5.5$ recently revealed a remarkable molecule harbouring a tunnel and homology modelling suggested a lipid transfer mechanism for the related molecules CD36 and SR-BI ${ }^{9}$. The most notable function of LIMP-2 is that it serves as a lysosomal sorting receptor for $\beta$-glucocerebrosidase $(\beta \text {-GCase })^{10}$, deficiency of which causes Gaucher disease, a lysosomal storage disease in which glucocerebrosides accumulate ${ }^{11}$. A short motif in the cytoplasmic tail of LIMP-2 has been thought to be solely responsible for lysosomal targeting ${ }^{10,12}$, making it a paradigm for mannose 6-phosphate (M6P) receptor (MPR)-independent lysosomal targetting. LIMP-2 binds to $\beta$-GCase in a $\mathrm{pH}$ dependent manner, and dissociation occurs in acidic late endosomal/lysosomal compartments. Here, by determining a structure at a different $\mathrm{pH}$, we are able to show the mechanism in atomic detail of $\mathrm{pH}$-dependent release of $\beta$-GCase. The new structure also shows that LIMP-2 is modified by the posttranslational addition of M6P, suggesting that it interacts with MPR. We show using biophysical methods and live cell microscopy that this interaction does occur and, by formation of a ternary complex of MPR, LIMP- 2 and $\beta$-GCase provides a subcellular targeting mechanism.

\section{Results}

Structure determination. Human LIMP-2 luminal domain containing residues $28-431$ was produced by stable expression in HEK293S (GnTI - ) cells and crystallized in two space groups, $C 222_{1}$ and $\mathrm{C2}$, at $\mathrm{pH}$ 6.5. X-ray diffraction data were collected at the Diamond Light Source, and structures of both crystal forms were determined using the molecular replacement method based on the model of LIMP-2 at lower $\mathrm{pH}^{9}$ and refined at $2.8 \AA$ resolution. The final refined models have reasonable $R$-factors and very good stereochemistry. Details of protein expression, purification, crystallization and structure determination are given in Methods section and Table 1.

Overall structure. Residues 38-429 of the bean-shaped molecule are visible, the structure fading out nine and two residues (at the $\mathrm{N}$ - and C-termini, respectively) from the terminal trans-membrane helices (Fig. 1a). The electron density maps for both space groups are of good quality with only 11 terminal residues disordered with 29 and 30 glycosidic units well ordered in the two space groups (Fig. 2 and Supplementary Table 1). Although sugars are seen on all nine potential sites, the electron density is too diffuse to permit modelling for the sugar attached to residue N105. The structure can be divided into three domains (Fig. 1a). Domain I, the core of the structure, comprises a 13-stranded antiparallel $\beta$-barrel wedged open at the membrane-proximal end by the insertion into the barrel of the $\beta 9-\beta 10$ loop. The narrower membrane-distal part of domain I (formed from the three longest strands: $\beta 1, \beta 16$ and $\beta 17)$ is flanked by two smaller domains, II and III. Domain II (residues 126-205), inserted between strands

\section{Table 1 | Data collection and refinement statistics.}

\begin{tabular}{|c|c|c|}
\hline \multicolumn{3}{|l|}{ Data collection } \\
\hline Space group & $C 222_{1}$ & $\mathrm{C} 2$ \\
\hline \multicolumn{3}{|l|}{ Cell dimensions } \\
\hline$a, b, c(\AA)$ & $63.7,95.4,217.8$ & $89.7,63.4,114.4$ \\
\hline$\alpha, \beta, \gamma\left({ }^{\circ}\right)$ & $90,90,90$ & $90,102.3,90$ \\
\hline Resolution $(\AA)$ & $50.0-2.80(2.90-2.80)^{\star}$ & $50.0-2.80(2.90-2.80)$ \\
\hline Unique reflections & $15,620(1,535)$ & $15,244(1,460)$ \\
\hline$R_{\text {merge }}$ & $0.180(0.636)$ & $0.119(0.378)$ \\
\hline$|/ \sigma|$ & $9.0(2.2)$ & $7.1(1.8)$ \\
\hline Completeness (\%) & $92.2(94.6)$ & $96.2(91.5)$ \\
\hline Redundancy & $6.6(5.0)$ & $2.5(2.1)$ \\
\hline \multicolumn{3}{|l|}{ Refinement } \\
\hline Resolution $(\AA)$ & $50.0-2.80$ & $50.0-2.80$ \\
\hline No. of reflections & 13,289/770 & $13,688 / 763$ \\
\hline$R_{\text {work }} / R_{\text {free }}$ & $0.222 / 0.283$ & $0.223 / 0.269$ \\
\hline \multicolumn{3}{|l|}{ No. of atoms } \\
\hline Protein & 3,128 & 3,148 \\
\hline Ligand/ion & 363 & 378 \\
\hline \multicolumn{3}{|l|}{ B-factors $\left(\AA^{2}\right)$} \\
\hline Protein & 45 & 53 \\
\hline Ligand/ion & 57 & 70 \\
\hline \multicolumn{3}{|c|}{ Root mean square deviations } \\
\hline Bond lengths $(\AA)$ & 0.005 & 0.005 \\
\hline Bond angles $\left(^{\circ}\right)$ & 1.2 & 1.3 \\
\hline
\end{tabular}

$\beta 5$ and $\beta 8$, and resting on the inner face of the three long strands of domain I, has two $\beta$-strands and four helices, three of which $(\alpha 4, \alpha 5$ and $\alpha 7$ ) form a bundle. Domain III (residues 297-365), inserted between $\beta 12$ and $\beta 15$, contains two loose coil regions separated by two short $\beta$-strands that pin the domain to $\beta 16$ of the $\beta$-barrel. Remarkably, a large tunnel extends through the centre of domain I from the membrane proximal end to near the top of the molecule, where it opens into a large cavity at the base of domain II, the domain to which $\beta$-GCase attaches (Fig. 3a) ${ }^{10}$. Similar tunnels in the related molecules SR-BI and CD36 act as a route for lipid transport ${ }^{9}$, but the role in LIMP-2 has remained obscure 9 . However, the residues lining the tunnel are highly conserved from zebrafish to man, suggesting that the function is preserved.

LIMP-2 possesses an N-linked P-Man ${ }_{9} G_{1 c N A c}$. Not only does the electron density map show that all nine potential $\mathrm{N}$-linked glycosylation sites in LIMP-2 bear sugars, but also that the density is sufficiently clear that at four positions five or more glycosides can be modelled. All these nine sites are conserved among different species apart from zebrafish, while the overall level of amino-acid identity is only $\sim 32 \%$ (ref. 9). The most surprising observation is that we can identify from the well-defined electron density that the sugar attached to N325 is P-Man ${ }_{9} \mathrm{GlNNAc}_{2}$ (Fig. 2), the first time this sugar, which bears a terminal M6P, has been seen in a protein structure. N325 is the last residue of $\beta 13$ and sits at the bottom of a cleft formed by $\alpha 4$ and the $\alpha 11-\beta 13$ loop (Fig. 1b). The first three saccharides of the glycan lie against a4 making mainly hydrophobic interactions on one side and ring-stacking interactions with a conserved cis-proline (P314) in the $\alpha 11-\beta 13$ loop, on the other side. The region around the N325 glycosylation site is stabilized by two conserved disulphides, C312-C318 within the $\alpha 11-\beta 13$ loop and C274-C329 that links the $\beta 13-\beta 14$ loop to the $\alpha 9-\beta 11$ loop. In the $C 222_{1}$ crystal, the 
a

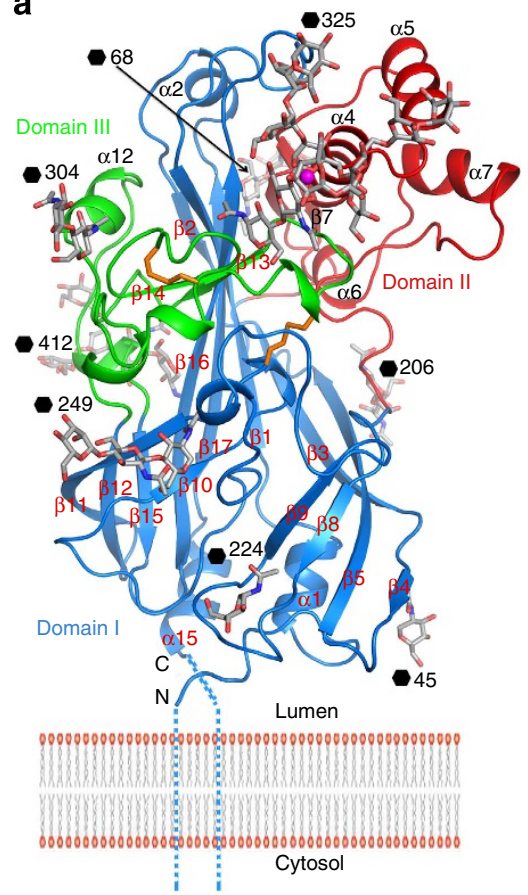

b

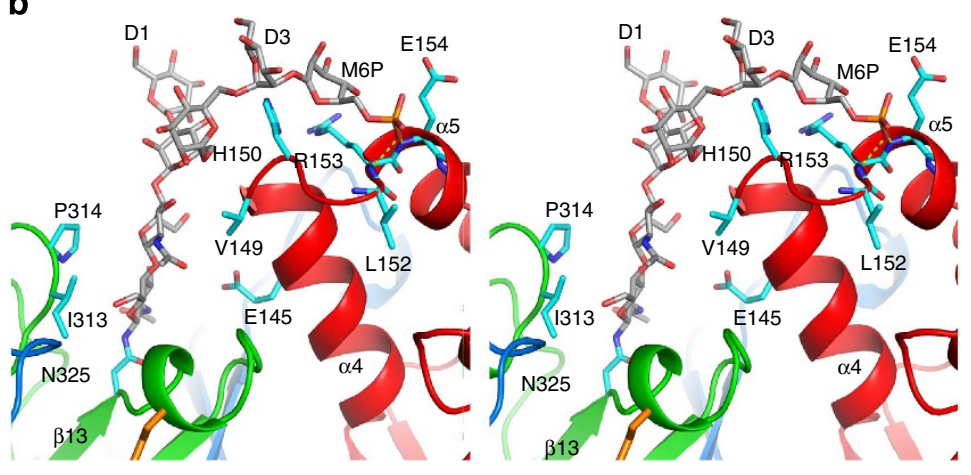

C

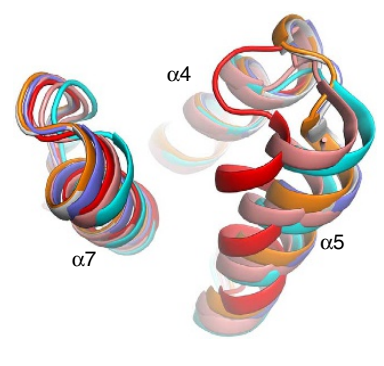

d

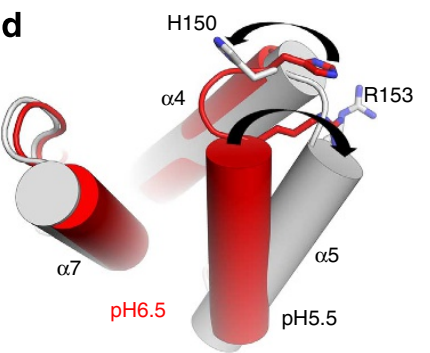

Figure 1 | Overall structure of LIMP-2 and structural differences at pH $\mathbf{6 . 5}$ and $\mathbf{5 . 5}$. (a) Ribbon diagram showing the overall structure of LIMP-2 with domains I-III coloured in blue, red and green, respectively. Disulphides are drawn as orange sticks. Secondary structures are named as in Neculai et al. ${ }^{9}$ (b) Stereo view of the N-linked P-Man ${ }_{9} \mathrm{GlcNAc}_{2}$ (grey sticks) at N325 in space group C2, and its interactions with the protein (side chains as cyan sticks). (c) Structural differences in the domain II helical bundle at $\mathrm{pH} 6.5$ (red) and those from six molecules at pH 5.5. (d) The switch in conformation of the helical bundle from $\mathrm{pH} 6.5$ (red) to $\mathrm{pH} 5.5$ (grey) is indicated by the black arrows.

sugars are also stabilized by interaction with residues from a symmetry-related molecule that pack directly at the origin of the three arms of the glycan, forming a network of hydrogen bonds (Supplementary Figs 1 and 2), although this destabilizes the distal phosphate group. A well-defined ion (likely $\mathrm{Na}^{+}$) bridges the $\mathrm{O} 2$ atom of the $\beta$-mannose to $\mathrm{O} 4$ of the terminal D2 mannose (glycan nomenclature is defined in Supplementary Fig. 1a). The D3 arm of the glycan arches over the side chains of H150 and R153 that cap helix $\alpha 4$. The phosphate attached to O6 of the terminal D3 mannose is stabilized in the C2 crystal form by capping the $\mathrm{N}$ terminus of helix $\alpha 5$ via hydrogen bonds to the amide groups of R153, E154 and I155 (Figs 1 and 2).

P-Man GlcNAc $_{2}$ is positioned to bind MPR. Lysosomal enzymes are generally modified with a $\mathrm{P}-\mathrm{Man}_{9} \mathrm{GlcNAc}_{2}$ that is recognized by the MPR for transport ${ }^{13}$. The LIMP-2 we used for structure determination was produced by stable expression in HEK293S cells (see Methods section). These cells are deficient in the enzyme $\beta 1,2-\mathrm{N}$-acetylglucosaminyltransferase I (GNTI) that is required to process complex sugars from $\mathrm{Man}_{5} \mathrm{GlcNAc}_{2}$ (ref. 14). We would therefore expect that most sugars will be of this type; however, the electron density at residue N325 demonstrates that this deficiency in the sugar processing is not a hindrance to proper $\mathrm{P}-\mathrm{Man}_{9} \mathrm{GlcNAc}_{2}$ production. We presume that LIMP-2 therefore harbours a structural motif, which is known to be common to many soluble lysozymal enzymes, recognized by GlcNac-1 phosphotransferase ${ }^{15}$, the enzyme that phosphorylates the immature sugar. The presence of M6P suggested to us that MPR might attach to LIMP-2 in order to transport $\beta$-GCase to the lysosome in a hetero-trimeric complex (Fig. 4). In support of this, mutation of the residue N325 by which the M6P is attached to LIMP-2 abrogates transport to the lysosome ${ }^{9}$. We have confirmed this using confocal fluorescence microscopy (Supplementary Fig. 3), and have demonstrated that this is unlikely to be due to protein mis-folding, since soluble protein bearing the N325Q mutation could still be secreted (Supplementary Fig. 3c). If an MPR does bind it could either be the $46-\mathrm{kDa}$ cation-dependent receptor that functions as a dimer, or the $300-\mathrm{kDa}$ cation-independent (CI) receptor that contains 15 structural homology repeats with two M6P binding sites ${ }^{16}$, both are type I membrane proteins. Given the large distance from the M6P to the membrane in LIMP-2 ( $\sim 70 \AA$ based on the structure shown in Fig. 1a), the longer CI-MPR would be the most likely co-transporter (Fig. 4b).

LIMP-2 binds CI-MPR via M6P and simultaneously binds $\beta$-GCase. To establish if LIMP-2 and CI-MPR do indeed interact, we performed surface plasmon resonance (SPR) analyses (see Methods section). Three ligands were immobilized in a CM5 chip in parallel, with biotinylated CI-MPR domains I-XV, biotinylated $\beta$-GCase and biotinylated LIMP- $2_{\text {lum }}$ attached to separate flow cells. Soluble LIMP-2 was then flowed over all cells. The first experiment used LIMP-2 produced from HEK293S (GNTI - ) cells, the same material used for crystallization, which therefore had limited processing of the sugars. This version of LIMP-2 bound tightly to both CI-MPR $(\mathrm{Kd} 2.2 \mu \mathrm{M})$ and $\beta$-GCase $(\mathrm{Kd}$ $1.4 \mu \mathrm{M}$ ), but not to LIMP-2 itself (Fig. 5a). When fully glycosylated LIMP-2 produced from HEK293T cells was used, an even higher affinity of $\mathrm{Kd} 0.97 \mu \mathrm{M}$ was achieved for CI-MPR (Fig. 5b). To test the glycosylation dependence of binding, we used endoglycosidase F1 to trim the glycans to a single $\mathrm{N}$-acetylglucosamine. The deglycosylated LIMP-2 lost its ability to bind CI-MPR, whereas it retained its ability to bind $\beta$-GCase (Fig. $5 \mathrm{c}$ ). To assess the specificity for M6P, we used M6P as a chemical competitor. In the presence of $2 \mathrm{mM}$ M6P, LIMP- 2 could not bind CI-MPR while binding to $\beta$-GCase was not affected 

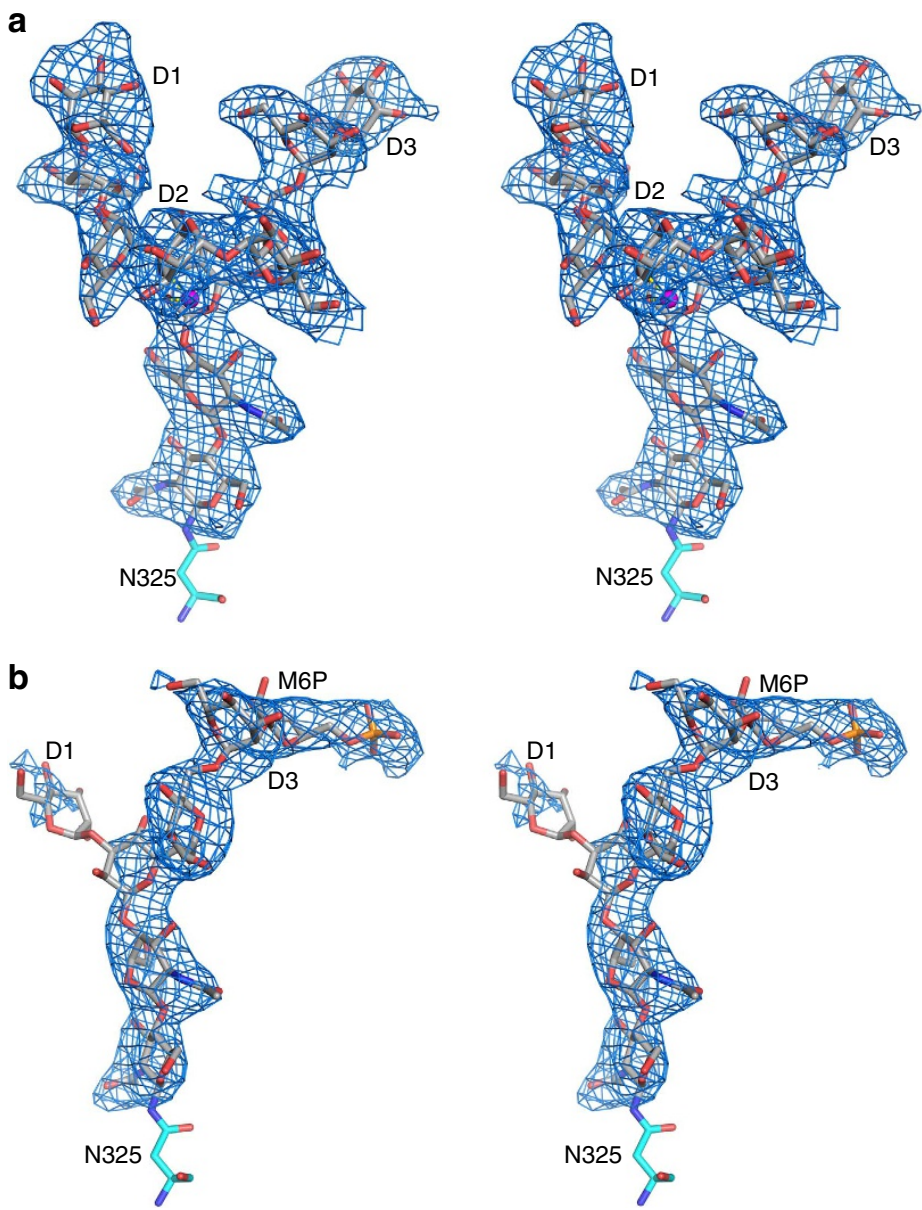

Figure 2 | Simulated annealing omit $\left|\boldsymbol{F}_{\mathbf{o}}-\boldsymbol{F}_{\mathbf{c}}\right|$ electron density map contoured at $\mathbf{2 . 5} \boldsymbol{\sigma}$. (a) Stereo diagram showing the well-defined electron density for the $\mathrm{N}$-linked $\mathrm{Man}_{9} \mathrm{GlcNAc}_{2}$ at N325 in space group $\mathrm{C}_{222}$. A Na ${ }^{+}$liganded by $\mathrm{O} 2$ of the $\beta$-mannose and $\mathrm{O} 4$ of the D2 mannose is shown as a magenta sphere. The phosphate at the D3 mannose is disordered. (b) The same glycosylation site in space group C2 showing the density for the M6P, but in this case residues in the D1 and D2 arms are disordered.

a

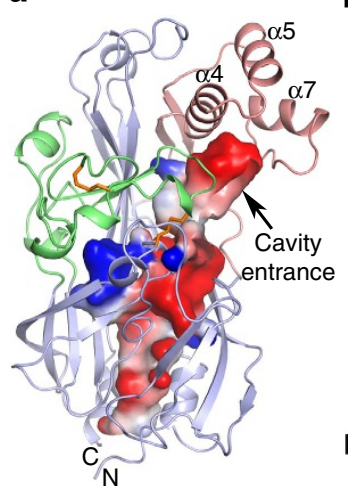

b

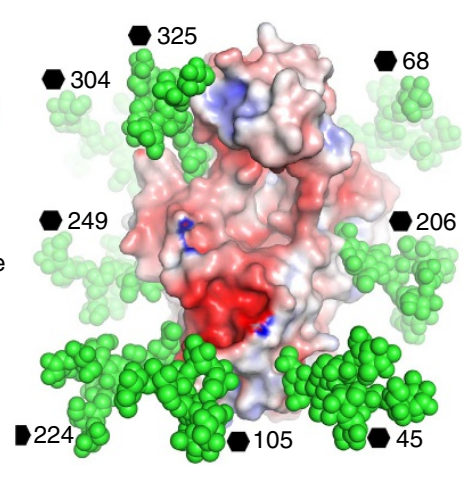

C

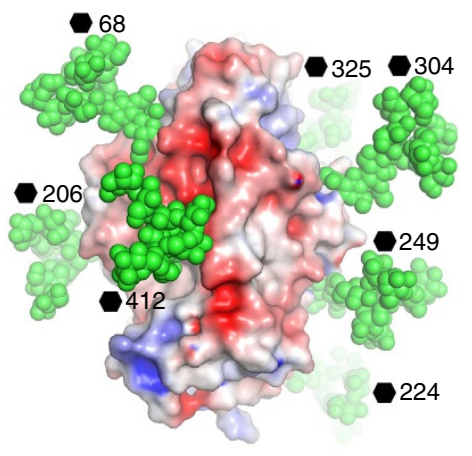

Figure 3 | Structural features of LIMP-2. (a) Electrostatic surface contoured at $\pm 5 \mathrm{kTe}^{-1}$ showing the tunnel and cavity in LIMP-2. The protein chain is shown as ribbons with its three domains coloured in pale blue, red and green, respectively. (b) Man, ${ }_{9} \mathrm{GlNAc}_{2}$ observed at N325 site is modelled at all nine glycosylation sites of LIMP-2 with atoms shown as green spheres on the electrostatic surface of the molecule to show glycan-free areas on the protein surface. (c) Approximately $180^{\circ}$ rotation of $\mathbf{b}$ showing the back of the molecule.

(Fig. 5d). We then competed simply with mannose to test the requirement for the 6-phosphate group. Mannose $(2 \mathrm{mM})$ did not affect LIMP-2 binding to CI-MPR (Fig. 5e). Finally, we tested with glucose-6-phosphate (G6P) to confirm that the binding is M6P specific. G6P (2 $\mathrm{mM})$ did not affect LIMP-2 binding to CI-MPR (Fig. 5f). We also tested LIMP-2 produced from
HEK293S GNTI - cells for CI-MPR binding in the presence of the above sugars (Supplementary Fig. 4) and confirmed that LIMP-2 with limited glycosylation behaves similarly to fully glycosylated LIMP-2. Those data clearly demonstrate that LIMP2 binds CI-MPR in a M6P-dependent manner. To test if $\beta$-GCase, LIMP-2 and MPR form a hetero-trimeric complex, we attached 
a

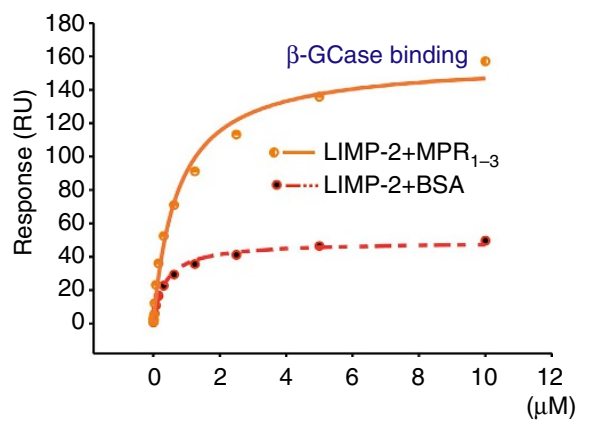

b

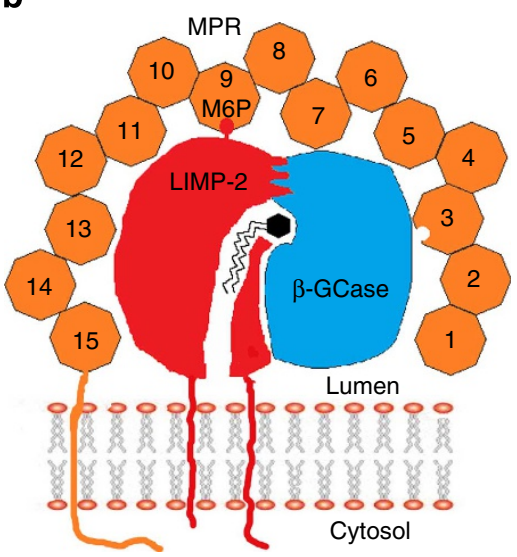

Figure 4 | LIMP-2-mediated interactions. (a) SPR experiment showing that $\beta$-GCase binds to a preformed LIMP-2/Cl-MPR complex to form a ternary complex, and also binds LIMP-2 alone (see also Fig. 5). $\beta$-GCase was biotinylated and attached to a CM5 chip, LIMP-2 mixed with MPR domains I-III in 1:1 ratio was then flowed over the chip. LIMP-2 mixed with BSA was used as a control. (b) A cartoon showing the complex of LIMP-2/ $\beta$-GCase/ CI-MPR. The orange octagons represent the 15 homologous domains of $\mathrm{Cl}-\mathrm{MPR}$ with its two M6P binding sites at domains III and IX. Which of the sites interacts with the M6P of LIMP-2 remains to be determined. The zig-zag lines with a hexagon head represent the glucocerebroside substrate of $\beta$-GCase. $\mathrm{RU}$, resonance units.

biotinylated $\beta$-GCase to a CM5 chip. LIMP-2 mixed with an equal amount (1:1 molecular ratio) of CI-MPR (domain I-III, with Rodopsin 1D4 tag, produced from HEK293S GNTI - cells, see Methods section), or same amount of LIMP-2 mixed with an equal amount of BSA, was flowed over the same chip. The results show that the LIMP-2/MPR has a much higher response than LIMP-2/BSA, demonstrating the presence of the hetero-trimeric $\beta$-GCase/LIMP-2/MPR complex (Fig. 4).

Detection of LIMP-2 and CI-MPR interaction in living cells. Since soluble LIMP-2 and CI-MPR bind specifically in vitro, we tested the interaction of the full-length membrane proteins in living cells by fluorescence lifetime imaging to detect Förster resonance energy transfer (FLIM-FRET). FLIM-FRET provides direct information about protein-protein interactions provided that appropriately labelled donor and acceptor molecules are positioned within $\sim 10 \mathrm{~nm}$ with the right orientation (this proximity shortens the donor fluorescence lifetime due to FRET) ${ }^{17}$. A monomeric teal fluorescent protein 1 (mTFP1, a FRET donor) ${ }^{18}$ was attached to LIMP-2 and mVenus (a FRET acceptor) ${ }^{19}$ was attached to CI-MPR (see Methods section). First, we examined, in COS 7 cells, subcellular localization of fluorescently labelled LIMP-2 and CI-MPR, with mouse Ras-related protein Rab5a-mCherry as marker for early endosomes ${ }^{20}$. The punctate co-localization of LIMP-2 with Rab5a-mCherry and LIMP-2 with CI-MPR is evident (Supplementary Fig. 3). When COS 7 cells co-expressed (via co-transfection) both LIMP2-mTFP1 and CI-MPR-mVenus fusion proteins, the average diminution of the donor mean lifetime $(\langle\tau\rangle)$ was $2.52 \pm 0.03 \mathrm{~ns}(n=11)$, significantly shorter (Student's $t$-test, $P=6.61 \times 10^{-6}$ ) than the donor alone $(\langle\tau\rangle$ ) $2.65 \pm 0.04 \mathrm{~ns}(n=12)$, indicating FRET between LIMP-2 and CI-MPR. A representative experiment is shown in Fig. 6. In this particular example, the average lifetime diminution in some pixels was calculated to be little as $2.32 \mathrm{~ns}$. We have also calculated the fraction of interacting donor $\left(f_{\mathrm{D}}\right)$ to quantify the extent of this interaction ${ }^{21,22}$. Indeed, $f_{\mathrm{D}}$ is an interesting parameter to characterize protein-protein interactions in living cells rather than the true FRET efficiency $(E)$ that defines the relative orientation of the donor relative to the acceptor ${ }^{17}$. In Fig. $6 \mathrm{~b}$, the average $f_{\mathrm{D}}$ coming from all pixels of the image was $\sim 0.25$. Observe that we also calculated the error for the cell expressing LIMP-2-mTFP1 alone $(\sim 0.05)$. However, when COS 7 cells were co-transfected with LIMP-2-N325Q-mTFP1 and CI-MPR-mVenus, the distribution of LIMP-2 within the cells became diffuse, whereas CI-MPR maintained the punctate pattern, and FLIM imaging no longer showed a significant mean lifetime diminution $(<\tau>=2.77 \mathrm{~ns})$ as compared with LIMP-2N325Q-mTFP1 expressed alone $(<\tau>=2.78 \mathrm{~ns})$ The corresponding $f_{\mathrm{D}}$ here are really close to $0(0.05$ in both cases), and show the absence of interaction between LIMP-2N325Q-mTFP1 and CI-MPR-mVenus (Fig. 7). When calculating these parameters for a population of cells (Fig. 8a), we found a mean of the average $f_{\mathrm{D}}$ of $0.17 \pm 0.03$ for LIMP-2mTFP1 and CI-MPR-mVenus interaction $(n=10$ from three independent experiments), and a mean of the average $f_{\mathrm{D}}$ of $0.05 \pm 0.01$ for LIMP-2-N325Q-mTFP1 and CI-MPR-mVenus $(n=10)$; this $f_{\mathrm{D}}$ corresponds to the error coming from the technique since a similar error was found for both LIMP-2N325Q-mTFP1 and LIMP-mTFP1 expressed alone (Fig. 8a). A $t$-test between the groups showed a statistically significant difference only for the difference between LIMP-2-mTFP1 alone and LIMP-2-mTFP1 in the presence of MPR-mVenus $\left(P=1.08 \times 10^{-4}\right)$. Importantly, when plotting the intensity ratio between the donor and the acceptor expression against the corresponding $f_{\mathrm{D}}$, we found a random distribution, confirming that the level of acceptor expression did not affect our results (Fig. 8b). These data demonstrate the in vivo interaction between LIMP-2 and CI-MPR, and suggest that the single N325Q mutation, which ablates the M6P attached to this residue, abolishes this interaction. We have shown above that this mutation does not prevent the secretion of the soluble version of this mutant, indicating correct folding.

Potential binding site for $\beta$-GCase. In order to highlight the possible $\beta$-GCase and CI-MPR binding areas of LIMP-2, we have modelled all N-linked glycans of LIMP-2 as $\mathrm{Man}_{9} \mathrm{GlcNAc}_{2}$ (as a crude model for complex sugars). A glycan-free belt remains, traversing from the front, over the top, to the back of the molecule (Fig. 3). Since extra glycan in an H363N mutant or lack of glycan in an N68Q mutant both impair LIMP-2 trafficking to the 
a

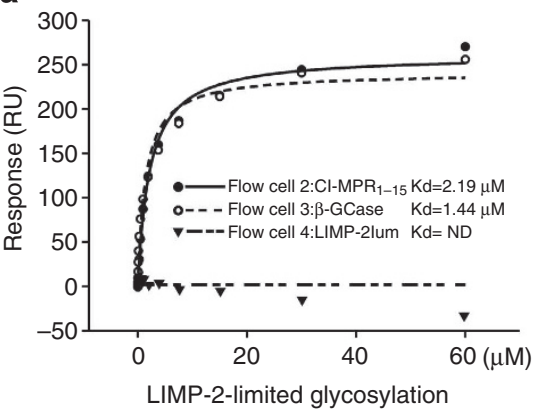

C

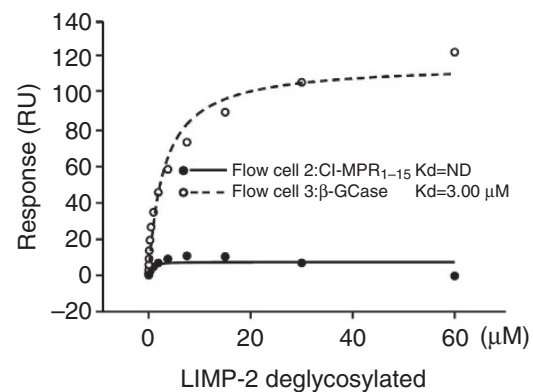

e

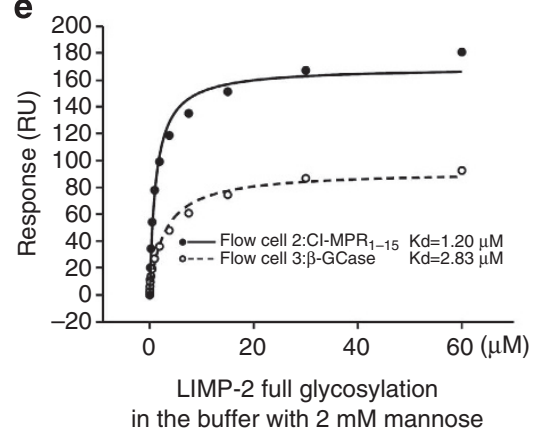

b

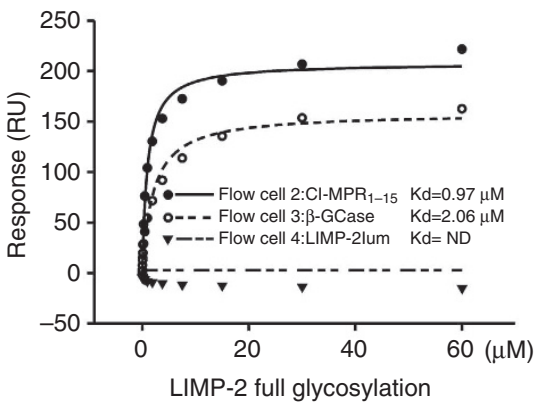

d

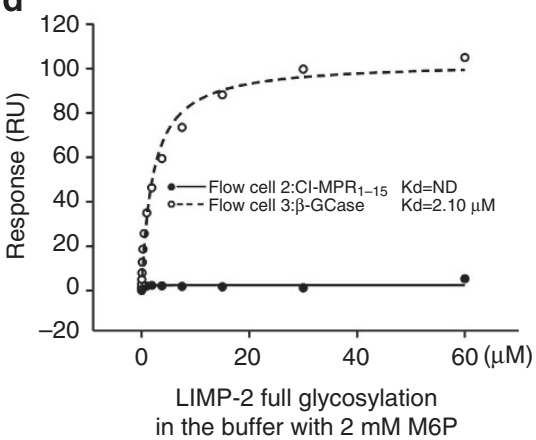

f

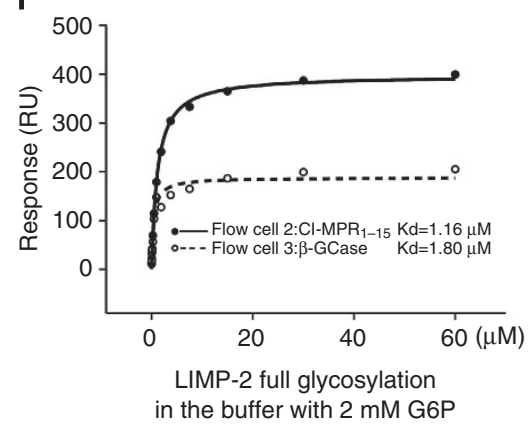

Figure 5 | SPR experiments for the interaction of LIMP-2 with CI-MPR and $\beta$-GCase. Biotinylated human CI-MPR domains I-XV and $\beta$-GCase were immobilized in flow cells 2 and 3 of a CM5 chip, respectively. LIMP-2 in different glycosylation status and in different sugar containing buffer was used as analyte. (a) LIMP-2 produced from HEK293S (GNTI - ) cells, with limited glycosylation. (b) LIMP-2 produced from HEK293T cells, with full glycosylation. (c) LIMP-2 produced from HEK293S (GNTI - ) cells and deglycosylated with endoglycosidase F1. (d) Fully glycosylated LIMP-2 in buffer containing 2 mM M6P. (e) Fully glycosylated LIMP-2 in buffer containing $2 \mathrm{mM}$ mannose. (f) Fully glycosylated LIMP-2 in buffer containing 2 mM G6P. ND, not detectable; $\mathrm{RU}$, resonance units.

lysosome ${ }^{9,23}$, and the former does not affect $\beta$-GCase binding, it is likely that MPR interacts with the back, and $\beta$-GCase binds to the front of LIMP-2 (Fig. 3). This provides a plausible explanation for the role of the tunnel in LIMP-2. In CD36 and SR-BI, this is used for lipid delivery, and if $\beta$-GCase binds with its active site facing the cavity of LIMP-2 then it could accommodate the long lipidic portion of the glucosylceramide substrate of $\beta$-GCase ${ }^{24}$. It is even possible that in the endosome, glucosylceramide is delivered from the inner leaflet of the membrane through the tunnel to the active site of $\beta$-GCase (Fig. $4 \mathrm{~b}$ ). This would imply that LIMP-2 acts as a regulatory subunit of $\beta$-GCase. We note, however, that it is also possible that the tunnel might harbour phosphatidylinositol or a derivative, as it has been established that phosphatidylinositol 4 kinases control lyososomal delivery of the LIMP-2/ $\beta$-GCase complex ${ }^{25}$.

Conformational changes in LIMP-2 mirror $\mathrm{pH}$ dependency of $\beta$-GCase binding. In the late endosome/lysosome $\beta$-GCase disassociates with the drop in $\mathrm{pH}$, and associates instead with saposin $\mathrm{C}$, which appears to increase the activity of the enzyme ${ }^{26}$. To understand this $\mathrm{pH}$-dependent dissociation, we compared our $\mathrm{pH} 6.5$ structures with the six independent molecules of the crystallographic structure at pH 5.5 (ref. 9). Although our two structures at $\mathrm{pH} 6.5$ are almost identical to each other (root mean squared deviation $=0.3 \AA$ for all $\mathrm{C} \alpha$ atoms), they differ significantly from the $\mathrm{pH} 5.5$ structures (root mean squared deviation $=0.5-0.6 \AA$ ), with the largest difference occurring in the helical bundle of domain II, which includes exactly the region (residues 150-167) identified as the $\beta$-GCase binding site (Fig. $1 \mathrm{c})^{10}$. This region shows $\mathrm{pH}$-dependent changes in flexibility as well as conformation. At $\mathrm{pH} 5.5$, the $\alpha 4-\alpha 5$ region shows considerable flexibility, the two-residue linker between $\alpha 4$ and $\alpha 5$ adopting two major conformations, three residues out of step with each other. In contrast, at $\mathrm{pH} \mathrm{6.5,} \mathrm{the} \mathrm{helical}$ bundle is very well defined and similar in both space groups. At higher $\mathrm{pH}$, the $\alpha 4-\alpha 5$ linker (residues 151-152) folds 


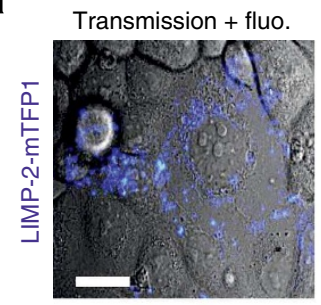

Number of photons
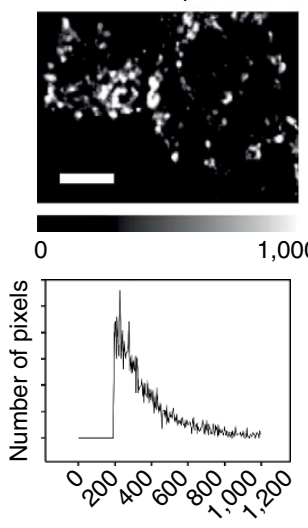

Number of photons

b

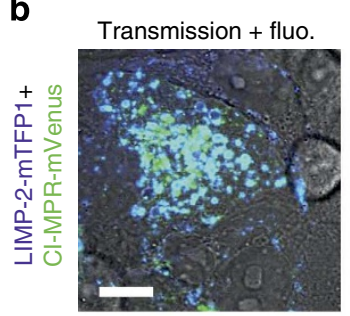

Number of photons
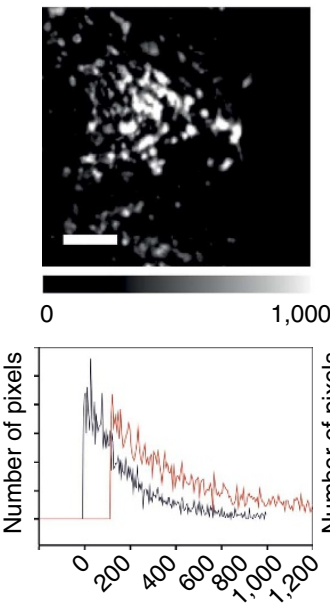

Number of photons
mTFP1 channel

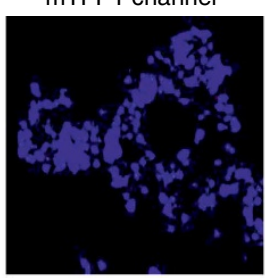

Mean lifetime (ns)

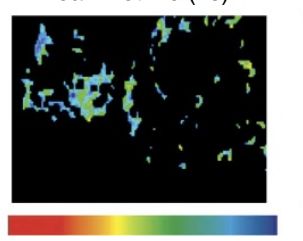

3.00 .0

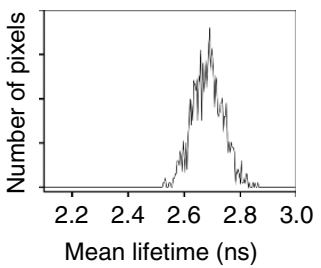

mTFP1 channel

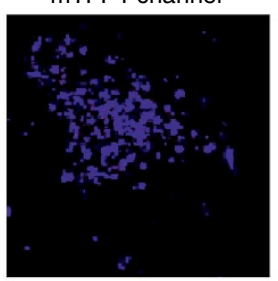

Mean lifetime (ns)

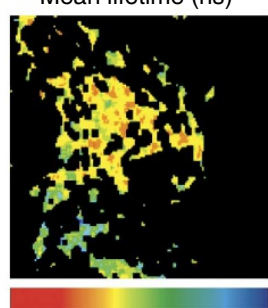

2.1

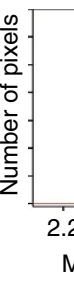

mVenus channel

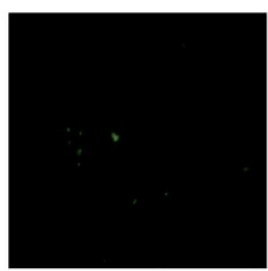

$f_{\mathrm{D}}$
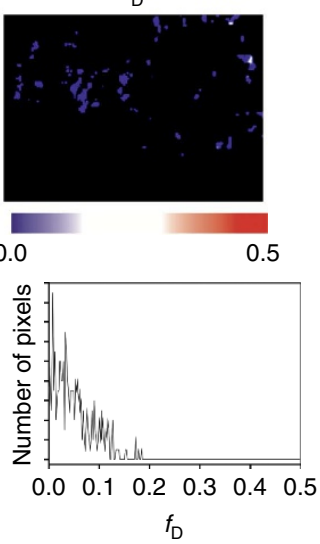

mVenus channel

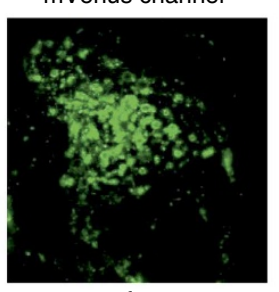

$f_{\mathrm{D}}$
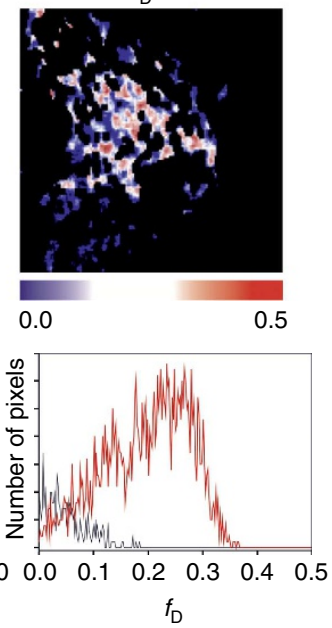

Figure 6 | FLIM-FRET between LIMP-2 and CI-MPR. (a) COS 7 cells expressing LIMP-2-mTFP1 alone (first row) show a punctate appearance (second panel from the left). The average number of acquired photons after applying a $2 \times 2$ spatial binning is 413 photons, the average mean lifetime ( $<\tau>$ ) for this sample is $2.68 \mathrm{~ns}$, and finally the average $f_{\mathrm{D}}$ is $5 \%$ (note in this and all subsequent panels, $f_{\mathrm{D}}$ corresponds to the fraction of LIMP-2 directly interacting with an acceptor). (b) COS-7 cells co-expressing LIMP-2-mTFP1 and CI-MPR-mVenus also show a punctate appearance and a high degree of colocalization (first row). The average number of photons after applying a $2 \times 2$ binning filter is $\sim 600$, the average mean lifetime ( $<\tau>$ ) is 2.52 ns, which indicates FRET, the mean $f_{\mathrm{D}}$ for this sample is $28 \%$. The average lifetime diminution is calculated in some pixels to be down to $2.32 \mathrm{~ns}$ (red pixels, second

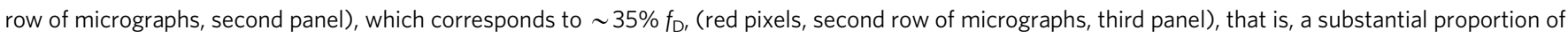
the LIMP-2 is involved in a direct interaction. fluo., fluorescent channel. Scale bar, $5 \mu \mathrm{m}$. 
a
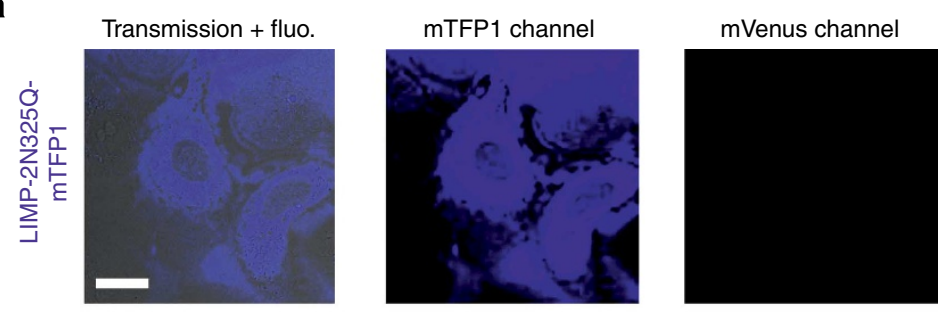

Number of photons

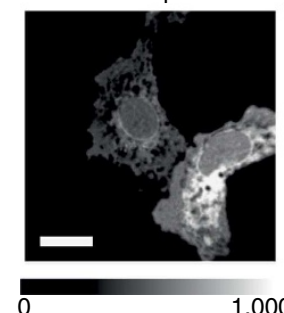

Mean lifetime (ns)
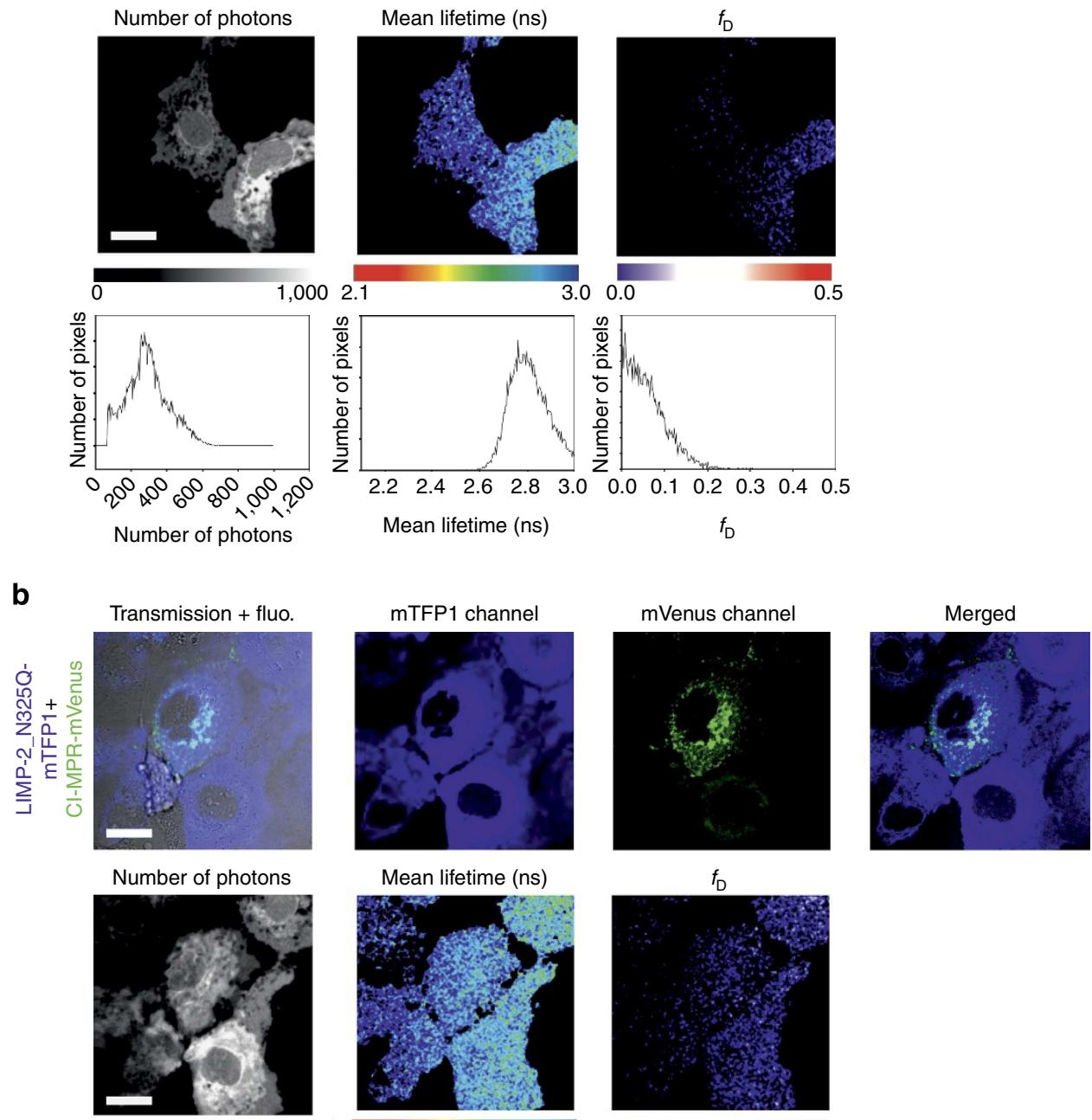

Mean lifetime (ns)

$f_{\mathrm{D}}$
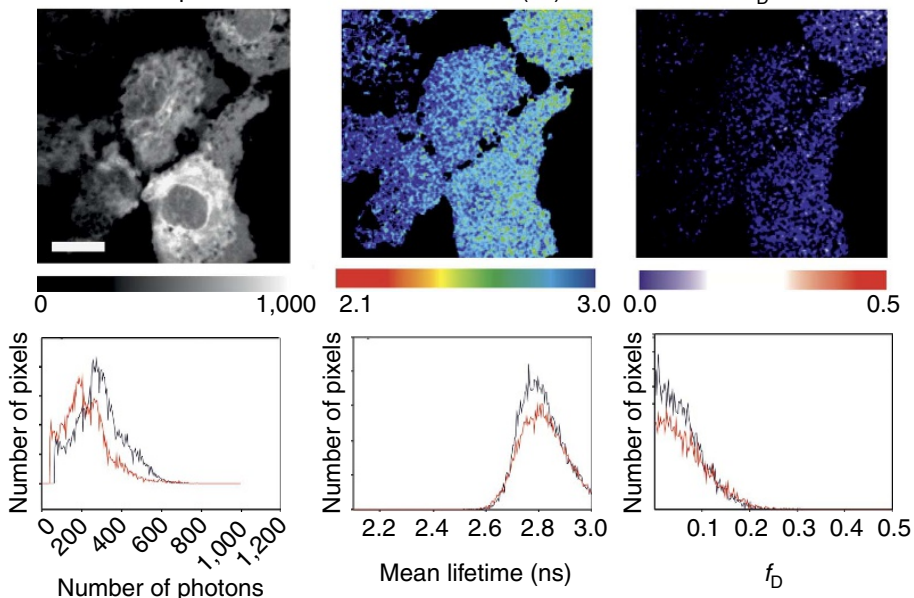

Figure 7 | LIMP-2-N325Q mutant and CI-MPR show no detectable FRET. (a) COS-7 cells expressing LIMP-2-N325Q-mTFP1 shows a diffuse pattern (first row). The average number of photons after applying a $2 \times 2$ spatial binning is $\sim 300$, the average mean lifetime is found to be $2.77 \mathrm{~ns}$ and the corresponding $f_{\mathrm{D}}$ of $6 \%$, that is, essentially no interaction, see Fig. 6 and main text (second row). (b) COS 7 cells co-expressing LIMP-2-N325Q-mTFP1 and CI-MPR-mVenus show a diffuse pattern for LIMP-2-N325Q-mTFP1 (blue, second panel from the left) and punctate pattern for CI-MPR-Venus (third panel from the left). The FLIM image (second row, second panel) does not show a significant mean lifetime diminution ( $\langle\tau\rangle=2.77 \mathrm{~ns}$ ), which is not significantly different from the control (LIMP-2-N325Q-mTFP1 alone), and hence no FRET is detected. Scale bar, $5 \mu \mathrm{m}$.

towards $\alpha 7$, such that $\alpha 5$ tilts about $20^{\circ}$ towards $\alpha 4$ and $\alpha 7$, making the bundle more compact and further stabilized by the C-terminal cap of $\alpha 4$ formed by His150 and Arg153 (Fig. 1b,d). At $\mathrm{pH} 6.5$, residues $152-163$ constitute $\alpha 5$, whereas at lower $\mathrm{pH}$ the helix starts either at 149 or 152 (Fig. 1c). Our structure, determined at $\mathrm{pH} 6.5$, similar to that of the endoplasmic reticulum (ER) and endosome, is likely to be competent to bind $\beta$-GCase. 


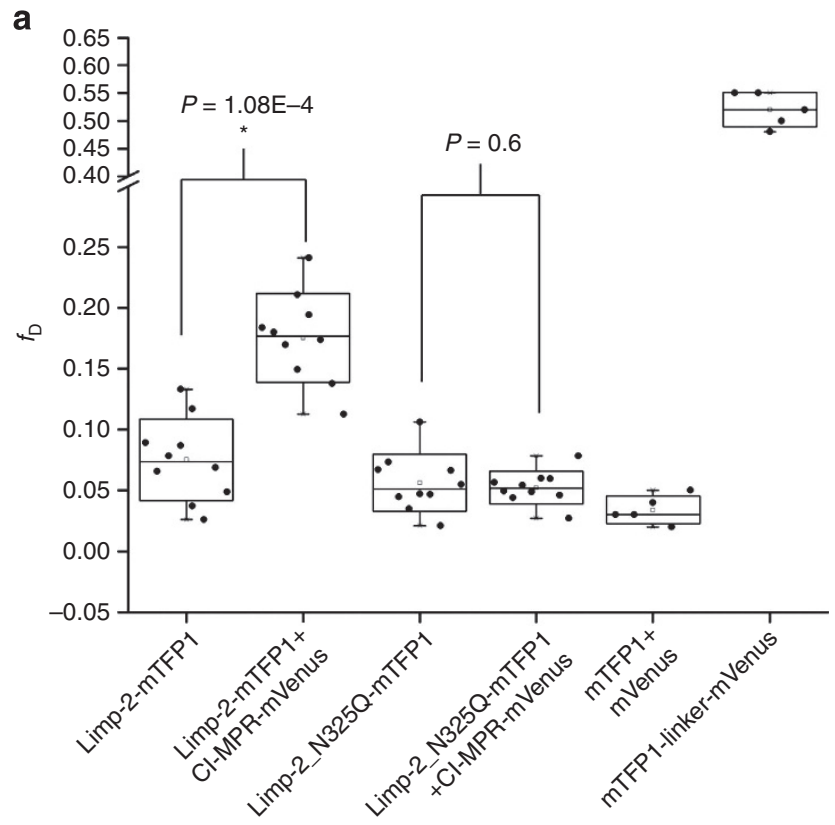

b

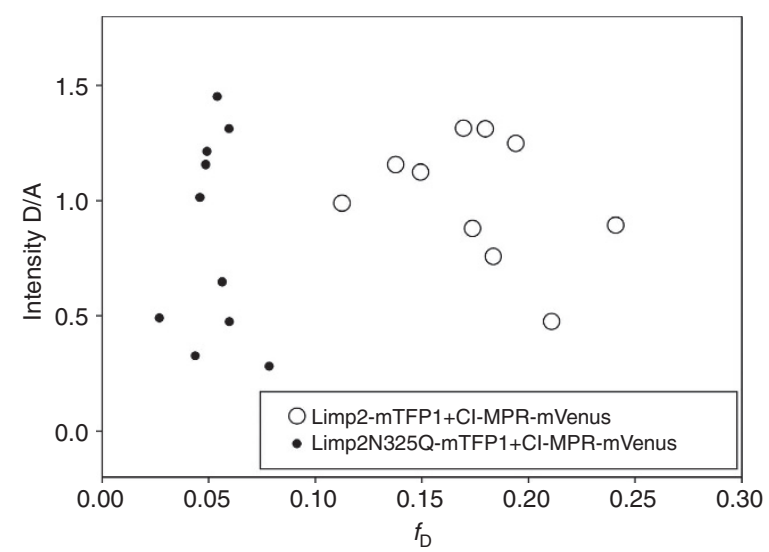

Figure 8 | Statistic analysis of the FLIM-FRET from LIMP-2 and CI-MPR.

(a) A box plot shows the mean (the box middle horizontal line), the s.d. (top and bottom of the box), the maximal and minimal values (whiskers) of $f_{D}$ distribution for LIMP-2-mTFP1 expressed alone $\left(f_{D}=0.07 \pm 0.03\right.$, $n=10)$ and LIMP-2-mTFP1 with CI-MPR-mVenus $\left(f_{D}=0.17 \pm 0.03, n=10\right.$ from three independent experiments). The $f_{\mathrm{D}}$ of the later is statistically significant compared with LIMP-2-mTFP1 alone $\left(P=1.8 \times 10^{-4}\right)$. The corresponding $f_{\mathrm{D}}$ values from LIMP-2-N325Q-mTFP1 expressed alone $(0.05 \pm 0.02, n=10)$ and LIMP-2-N325Q-mTFP1 with CI-MPR-mVenus $(0.05 \pm 0.01)$ show no statistical difference $(P=0.6)$. The fluorescent protein only donor (mTFP1) co-expressed with the acceptor (mVenus) is used as negative control while the donor (mTFP1) linked the acceptor (mVenus) with a flexible polypeptide chain of 13 amino acids is used as a positive control. The values were calculated using Sigma Plot (Student's $t$-test). (b) Scatter plot showing the distribution of averaged mean $f_{D}$ values against their corresponding donor and acceptor intensity ratio. A random distribution in both cases shows $f_{D}$ values are independent of acceptor intensity.

Histidine 150 acts as a $\mathbf{p H}$ sensor. $\mathrm{H} 171$ has been proposed to act as a pH sensor ${ }^{27}$, however, it is located on $\beta 7$ at the base of domain II (Supplementary Fig. 5), hydrogen bonds to E175 $(3.2 \AA)$ and does not undergo noticeable conformational change with $\mathrm{pH}$. In contrast, $\mathrm{H} 150$ caps $\alpha 4$ at higher $\mathrm{pH}$ and seems more likely to act as the trigger for the markedly altered conformation of $\alpha 4$ and $\alpha 5$. At lower $\mathrm{pH}, \mathrm{H} 150$ and
R153 move away, decapping the $\alpha 4 \mathrm{C}$ terminus and triggering a cascade of structural change in the helical bundle that leads to the dissociation of $\beta$-GCase from LIMP-2. To establish if $\mathrm{H} 150$ is a $\mathrm{pH}$ sensor, we performed SPR experiments of $\beta$-GCase binding to H150T mutant LIMP-2 and used wild-type LIMP-2 as a control. The experiments clearly show that wild-type LIMP-2 binds $\beta$-GCase at $\mathrm{pH} 6.5$, but not at $\mathrm{pH} 5.5$, whereas the H150T mutant LIMP-2 binds $\beta$-GCase at both pH 6.5 and 5.5 (Fig. 9). The results suggest that the H150T mutant is locked in the $\beta$-GCase binding conformation seen in the higher $\mathrm{pH}$ structure and unable to switch conformation at lower $\mathrm{pH}$, demonstrating that $\mathrm{H} 150$ is indeed a $\mathrm{pH}$ sensor.

\section{Discussion}

In summary, our structures illuminate several aspects of the mechanism of $\beta$-GCase transport, indeed the location of a putative lipid transport channel suggests that LIMP-2 may act not only as a transporter but also as a regulatory subunit, analogous to the activity-enhancing saposin $\mathrm{C}$ that binds $\beta$-GCase following its release from LIMP-2 (ref. 26). In addition, LIMP-2 is a receptor for EV71 and Coxsackievirus A16 (refs 28-30), and residues 144-151, the C-terminal half of $\alpha 4$, are supposed to interact directly with the virus ${ }^{28,29}$. Since these residues are partially sheltered by a $\mathrm{P}-\mathrm{Man}_{9} \mathrm{GlcNAc}_{2}$ attached to residue N325, glycan may serve as an attachment point for EV71 and CAV16 infection.

Following the observation of a P-Man ${ }_{9} \mathrm{GlCNA}_{2}$ moiety attached to LIMP-2, we demonstrated that LIMP-2 binds CI-MPR via M6P, the same mechanism used for classical lysosomal hydrolase transport. It is conceivable that further phosphorylated sugar may be attached elsewhere on the molecule, although we see no evidence for it. Although the affinity between LIMP-2 and CI-MPR is lower than that reported between a soluble lysosomal enzyme, $\beta$-glucuronidase and CI-MPR ${ }^{31}$, we have shown by SPR that the affinity of LIMP-2 for CI-MPR is similar to its affinity for $\beta$-GCase, and that these three proteins form hetero-trimeric complexes in vitro. It seems that MPR contributes indirectly to lysosomal targeting of $\beta$-GCase by binding the LIMP-2 component of the $\beta$-GCase/LIMP-2 complex. This is corroborated by FLIM-FRET results, demonstrating that LIMP2 and CI-MPR are in close proximity in cells, while this co-localization is abolished for the mutant LIMP-2 lacking the M6P. This strongly suggests a direct interaction of the M6P with CI-MPR, however, it is conceivable that the asparagine mutation has an indirect effect. We note that while MPRs are essential in sorting a repertoire of over 60 different soluble acid hydrolases, this is only the second example of an MPR binding a membrane protein (CD26 binds CI-MPR, contributing to T-cell activation $^{32}$ ). Our results contrast with the report that $\beta$-GCase targeting is MPR independent ${ }^{10}$, which found that $\beta$-GCase targeting is maintained in the presence of a mutation to GlcNac-1 phosphotransferase that traps the enzyme in the endoplasmic reticulum and abolishes the lysosomal targeting of soluble enzymes $^{33}$. It may be that the mechanism of sorting is more complex and involves both MPR-dependent and MPRindependent processes.

Our results have also shown that the $\mathrm{pH}$-dependent binding of $\beta$-GCase to LIMP-2 is related to conformational changes in the domain II helical bundle. We propose that H150, which caps the $\mathrm{C}$ terminus of $\alpha 4$, acts as a $\mathrm{pH}$ sensor that is in contrast to a previous report that $\mathrm{H} 171$ may function as a critical $\mathrm{pH}$ sensor ${ }^{27}$. We demonstrate that mutation of $\mathrm{H} 150$ to a threonine locks LIMP-2 in the high $\mathrm{pH}$ conformation, which binds $\beta$-GCase at both $\mathrm{pH} 6.5$ and 5.5. It seems likely, therefore, that protonation of 

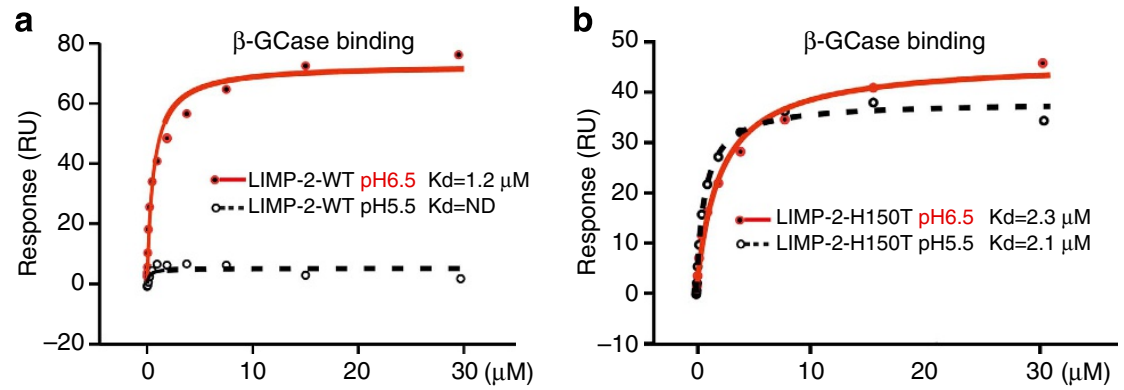

Figure 9 | Interactions of $\beta$-GCase with wild-type and H150T mutant LIMP-2. SPR experiments showing binding of $\beta$-GCase to (a) wild-type LIMP-2 and (b) H150T mutant LIMP-2 at pH 5.5 and pH 6.5. Biotinylated $\beta$-GCase was immobilized on a CM5 chip. Wild-type and H150T mutant LIMP-2 were used as analyte in phosphate citrate buffer. ND, not detectable; RU, resonance units.

$\mathrm{H} 150$ at lower $\mathrm{pH}$, for example, in the late endosome/lysosome environment, triggers conformational changes in the helical bundle and release of $\beta$-GCase from LIMP-2. However, since $\mathrm{H} 150$ is not conserved, it is possible that the mechanism is different in other species.

\begin{abstract}
Methods
Protein production. Protein production used a variant on our standard mammalian production system ${ }^{34}$, modified for the stable expression of a secreted product. All the PCR amplifications were carried out with KOD hot start DNA polymerase (EMD Millipore) according to manufacturer's recommendation (primers are listed in the Supplementary Table 2). Human LIMP-2 (UniProtKB/ Swiss-Prot Q14108) luminal domain (residues V28-T431) was PCR amplified from IMAGE clone 3872778 (BioScience) and cloned into a newly made in-house stable cell line vector pNeoSec (Supplementary Fig. 6). HEK293S GnTI ( - ) cells were co-transfected with a pNeoSec-LIMP-2 and a PhiC31 integrase expression vector (pCB92/pgk- $\phi$ C31; ref. 35). The polyclonal population resulting from G418 $\left(1 \mathrm{mg} \mathrm{ml}^{-1}\right)$ selection was cultured in Hyperflasks (Corning) in a CompacT SelecT-automated cell culture system (TAP Biosystems) ${ }^{34}$. The conditioned medium was dialysed and protein was purified with Talon $\mathrm{Co}^{2+}$ affinity resin (Clontech), and polished on a Superdex $20016 / 60$ column, eluted in $10 \mathrm{mM}$ HEPES, $\mathrm{pH} 7.4$, and $150 \mathrm{mM} \mathrm{NaCl}$. To produce fully glycosylated LIMP-2, the same construct was transiently transfected into HEK293T (ATCC CRL-11268) cells and protein purified in the same way. CI-MPR (UniProtKB/Swiss-Prot P11717) domains I-III (residue Q41-L489) was cloned into pURD vector in frame with a Rhodopsin 1D4 tag ${ }^{36}$. A stable HEK293S GNTI - cell line was generated by puromycin selection, and protein was purified as reported previously ${ }^{36}$.
\end{abstract}

Crystallization. LIMP-2 produced in HEK293S (GNTI - ) cells was concentrated to $4 \mathrm{mg} \mathrm{ml}^{-1}$, and crystallization screening was carried out using the sitting-drop vapour diffusion method in 96-well plates ${ }^{37}$. Images were taken using a TAP Biosystems storage vault $t^{38}$ at $21^{\circ} \mathrm{C}$. Clusters of needles appeared in $20 \%(\mathrm{w} / \mathrm{v})$ polyethylene glycol $3350,0.1 \mathrm{M}$ Bis-Tris propane, $\mathrm{pH} 6.5$, and $0.2 \mathrm{M}$ sodium acetate. Crystals were then crushed and cross-seeded into a set of crystallization screen conditions. The best diffracting crystals were obtained in $30 \%(\mathrm{w} / \mathrm{v})$ polyethylene glycol $8000,0.2 \mathrm{M}$ ammonium sulphate and $0.1 \mathrm{M}$ sodium cacodylate at $\mathrm{pH} 6.5$.

Data collection and structure determination. Crystals were flash frozen by immersion in a reservoir solution supplemented with $25 \%$ (v/v) glycerol followed by transfer to liquid nitrogen, and kept at $-173^{\circ} \mathrm{C}$ during X-ray data collection at I24, Diamond Light Source. Data images (exposure time $0.1 \mathrm{~s}$ with $30 \%$ beam transmission) of $0.2^{\circ}$ rotation were recorded on a PILATUS $6 \mathrm{M}$ detector, at a wavelength of $0.9686 \AA$. Data images were indexed and integrated with HKL2000, and reflections were merged with SCALEPACK ${ }^{39}$. The two crystal forms diffracted to $2.8 \AA$ resolution, with space groups of $C 222_{1}$ and $C 2$. Structure determination by molecular replacement with a LIMP-2 search model (PDB ID 4F7B) ${ }^{9}$ used

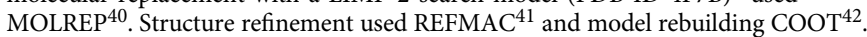
Table 1 shows data collection and refinement statistics. Figures were prepared using PyMOL ${ }^{43}$.

SPR equilibrium binding studies. Human $\beta$-GCase residues (40A-536R), human CI-MPR domains I-XV (residues 41Q-G2293) and human LIMP-2 luminal domain (residue V28-T431) were cloned into pHL-Avitag3 (ref. 44) and in vivo biotinylated by co-transfection with a BirA-ER plasmid ${ }^{45}$ into HEK293T cells. About 1,000 resonance units of each of the biotinylated proteins were immobilized on a CM5 sensor chip (GE Healthcare Life Sciences) to which streptavidin had been covalently coupled. We used a Biocore T100 machine (GE Healthcare) at $25^{\circ} \mathrm{C}$ with a running buffer comprising $10 \mathrm{mM}$ HEPES, $\mathrm{pH} 7.5,150 \mathrm{mM} \mathrm{NaCl}$ and $0.005 \%$ Tween 20 . The response was plotted versus the concentration of the analytes and fitted by nonlinear regression to a one-site saturation binding model (Sigma Plot, Systat software, Inc. San Jose, CA). For inhibition experiments, $2 \mathrm{mM}$ M6P, $2 \mathrm{mM}$ G6P or $2 \mathrm{mM}$ mannose was included in the above running buffer. For $\mathrm{pH}$-dependent binding, phosphate citrate buffer at either $\mathrm{pH} 6.5$ or 5.5 with $150 \mathrm{mM} \mathrm{NaCl}$ and $0.005 \%$ Tween 20 was used.

FLIM-FRET. Full-length human CI-MPR (residues Q41-I2491) was tagged at its C-terminal end in-frame with mVenus ${ }^{19}$ in a pHL-Sec-based vector ${ }^{4}$. Full-length human LIMP-2 (residues M1-T478) was tagged at its C-terminal end with mTFP-1 (ref. 18), and mouse Rab5a-mCherry was obtained from Addgene ${ }^{46}$ deposited by Christien Merrifield. The LIMP-2 N325Q mutation was made by a two-step overlapping PCR strategy and tagged with mTFP1 in the same way as the wild-type protein. COS 7 cells transiently co-expressing LIMP-2-mTFP1, CI-MPR-mVenus and Rab5a-mCherry, LIMP-2-mTFP1 and CI-MPR-mVenus, LIMP-2-N325QmTFP1 and CI-MPR-Venus or LIMP-2-mTFP1 (donor only, negative control) were grown to $60-70 \%$ confluence on glass-bottom $35 \mathrm{~mm}$ Petri dishes (Mattek) and imaged in phenol red-free medium. Multicolour images were acquired using a Leica SP8-X-SMD confocal microscope (Leica Microsystems, Manheim, Germany) with a $\times 63 / 1.4$ numerical aperture oil immersion objective. LIMP-2-mTFP1 and CI-MPR-mVenus were excited with a 440-nm laser and a white light laser (WLL) tuned to $514 \mathrm{~nm}$, respectively. Rab5a-mCherry was excited with the WLL tuned to $594 \mathrm{~nm}$. Fluorescence emission for mTFP1 and mVenus-labelled proteins was detected with two HyD detectors capable of photon counting; the mCherry fluorescence was detected using a cooled photomultiplier tube. The emission windows for the fluorescent proteins utilized were selected as follows: mTFP1 $(460-500 \mathrm{~nm})$, mVenus $(520-560 \mathrm{~nm})$ and mCherry $(600-650 \mathrm{~nm})$. The pinhole was set at one Airy unit.

FRET was detected and quantified by lifetime imaging (FLIM) using a (timedomain) time correlated single photon counting approach. The inverted laser scanning microscope Leica SP8-SMD equipped with a 440-nm pulsed laser (Picoquant $\mathrm{GmbH}$, Germany) tuned at $40 \mathrm{MHz}$ and single-photon counting electronics (PicoHarp 300) was used to excite the donor alone (LIMP-2-mTFP1) and the donor in the presence of acceptor (LIMP-2-mTFP1 + MPR-mVenus). The emitted blue photons (donor only) passed through a 460-500-nm filter and were detected with a HyD detector (Leica Microsytems). The acquisition times ranged from 2 to $3 \mathrm{~min}$ and at least 150 photons per pixel were collected in all cases. A $2 \times 2$ spatial binning was applied in all images to increase the signal to noise. The acquired fluorescence decays coming from regions of interest comprising one whole cell were deconvoluted with the instrument response function and fitted by a Marquandt nonlinear least-square algorithm using Symphotime software (Picoquant) with one- or two-exponential theoretical models. If FRET occurred the mean lifetime ${ }^{21}$ was shortened and a two species model (an interacting fraction corresponding to a population that relaxes through FRET $\left(f_{\mathrm{D}}\right)$ and a noninteracting fraction in which the donor lifetime remains undisturbed $\left.\left(1-f_{\mathrm{D}}\right)\right)$ was applied. The donor lifetime obtained from a single exponential fit from cells $(\sim 2.65 \mathrm{~ns})$ expressing the donor alone was used for the non-interacting fraction of the double exponential model in the corresponding co-transfected cell ${ }^{12,47,48}$.

$$
i(t)=\left(1-f_{\mathrm{D}}\right) e^{-t / \tau_{\mathrm{D}}}+f_{\mathrm{D}} e^{-t / \tau_{\mathrm{F}}}
$$

where $\tau_{\mathrm{D}}$ is the fixed donor lifetime and $\tau_{\mathrm{F}}$ is the discrete FRET lifetime. In this analysis, the total intensity $\left(I_{0}\right)$ was normalized to 1 and so that the pre-exponential factors lie in the range $0-1$ (Fig. 8).

The true FRET efficiency $(E)$ was calculated applying the formula:

$$
E=1-\tau_{\mathrm{F}} / \tau_{\mathrm{D}}=R_{0}^{6} / R_{0}^{6}+r^{6}
$$

where $\tau_{\mathrm{F}}$ is the FRET lifetime and $\tau_{\mathrm{D}}$ is the donor lifetime; $R_{0}$ is the Förster radius and $r$ is the distance between the donor and the acceptor. The $R_{0}$ for the mTFP1/ Venus was calculated as in Padilla-Parra et al. ${ }^{21}$, and was $57 \AA$ as reported in $\mathrm{Ai}$ 
et al. ${ }^{49}$ The average $E$ was found to be $0.69 \pm 0.06$ and the average distance between the two fluorescent proteins tagged to LIMP- 2 and MPR was $49 \pm 2.5 \AA$. Note that the true FRET efficiency $(E)$ is quite constant for all experiments $(n=11)$, whereas $f_{\mathrm{D}}$ varied depending on the extent of the interaction (Fig. 8); this calculation highlights the importance of recovering $f_{\mathrm{D}}$

The pixel by pixel $f_{\mathrm{D}}$ was obtained calculating the mean lifetime $\langle\tau\rangle$ using the first 1,200 channels ( 20 ps per channel) of the fluorescence decay in a pixel by pixel manner, a binning of $2 \times 2$ was applied to increase the signal to noise, although the mean lifetime is a parameter that is quite robust with just $\sim 100$ photons per pixel $^{50}$.

$$
\langle\tau\rangle=\int t \cdot i(t) \mathrm{d} t / \int i(t) \mathrm{d} t=\sum \Delta t_{i} \cdot I_{i} / \sum I_{i}
$$

In order to obtain $f_{\mathrm{D}}$, these images were then treated with ImageJ (http:// rsbweb.nih.gov/ij/) applying the formula:

$$
f_{\mathrm{D}}=\left[1-\langle\tau\rangle / \tau_{\mathrm{D}}\right] /\left[1-\langle\tau\rangle / \tau_{\mathrm{D}}-\left(\tau_{\mathrm{F}} / \tau_{\mathrm{D}}\right)^{2}+\left(\langle\tau\rangle / \tau_{\mathrm{D}}\right) \cdot\left(\tau_{\mathrm{F}} / \tau_{\mathrm{D}}\right)\right]
$$

where $\langle\tau\rangle$ is the mean lifetime, $\tau_{\mathrm{D}}$ is the fixed donor lifetime and $\tau_{\mathrm{F}}(\sim 0.83 \mathrm{~ns})$ is the fixed discrete FRET lifetime obtained from fitting (see above). The $f_{\mathrm{D}}$ plots together with the statistics ( $t$-tests) where calculated using Sigma Plot.

Western blot analyses of secreted LIMP-2 luminal domain. Wild-type and mutant domains were cloned into a pNeoSec vector with a C-terminal His tag and expressed in HEK293T cells for 2 days. Protein samples (conditioned media (M) or cell lysate (C)) were reduced (5 mM DTT) and separated by SDS-PAGE gel. Molecular weight markers (BenchMark) were used (Invitrogen). Western blots were probed with anti-His tag (Penta His, Qiagen), followed by goat anti-mouse IgG-horseradish peroxidise conjugate (Sigma). The enzymatic activity of horseradish peroxidise was detected using the Amersham ECL Western blotting detection kit (GE Healthcare Life Sciences).

\section{References}

1. Calvo, D., Dopazo, J. \& Vega, M. A. The CD36, CLA-1 (CD36L1), and LIMPII (CD36L2) gene family: cellular distribution, chromosomal location, and genetic evolution. Genomics 25, 100-106 (1995).

2. Fujita, H. et al. Isolation and sequencing of a cDNA clone encoding the $85 \mathrm{kDa}$ human lysosomal sialoglycoprotein (hLGP85) in human metastatic pancreas islet tumor cells. Biochem. Biophys. Res. Commun. 184, 604-611 (1992).

3. Chen, R. et al. Glycoproteomics analysis of human liver tissue by combination of multiple enzyme digestion and hydrazide chemistry. J. Proteome. Res. 8, 651-661 (2009).

4. Berkovic, S. F. et al. Array-based gene discovery with three unrelated subjects shows SCARB2/LIMP-2 deficiency causes myoclonus epilepsy and glomerulosclerosis. Am. J. Hum. Genet. 82, 673-684 (2008).

5. Rubboli, G. et al. Clinical and neurophysiologic features of progressive myoclonus epilepsy without renal failure caused by SCARB2 mutations. Epilepsia 52, 2356-2363 (2011).

6. Febbraio, M., Hajjar, D. P. \& Silverstein, R. L. CD36: a class B scavenger receptor involved in angiogenesis, atherosclerosis, inflammation, and lipid metabolism. J. Clin. Invest. 108, 785-791 (2001).

7. Canton, J., Neculai, D. \& Grinstein, S. Scavenger receptors in homeostasis and immunity. Nat. Rev. Immunol. 13, 621-634 (2013).

8. Yamayoshi, S. et al. Scavenger receptor B2 is a cellular receptor for enterovirus 71. Nat. Med. 15, 798-801 (2009).

9. Neculai, D. et al. Structure of LIMP-2 provides functional insights with implications for SR-BI and CD36. Nature 504, 172-176 (2013).

10. Reczek, D. et al. LIMP-2 is a receptor for lysosomal mannose-6-phosphateindependent targeting of beta-glucocerebrosidase. Cell 131, 770-783 (2007).

11. Jmoudiak, M. \& Futerman, A. H. Gaucher disease: pathological mechanisms and modern management. Br. J. Haematol. 129, 178-188 (2005).

12. Honing, S., Sandoval, I. V. \& von Figura, K. A di-leucine-based motif in the cytoplasmic tail of LIMP-II and tyrosinase mediates selective binding of AP-3. EMBO J. 17, 1304-1314 (1998).

13. Kim, J. J., Olson, L. J. \& Dahms, N. M. Carbohydrate recognition by the mannose-6-phosphate receptors. Curr. Opin. Struct. Biol. 19, 534-542 (2009).

14. Reeves, P. J., Callewaert, N., Contreras, R. \& Khorana, H. G. Structure and function in rhodopsin: high-level expression of rhodopsin with restricted and homogeneous $\mathrm{N}$-glycosylation by a tetracycline-inducible $\mathrm{N}$-acetylglucosaminyltransferase I-negative HEK293S stable mammalian cell line. Proc. Natl Acad. Sci. USA 99, 13419-13424 (2002).

15. Tikkanen, R., Peltola, M., Oinonen, C., Rouvinen, J. \& Peltonen, L. Several cooperating binding sites mediate the interaction of a lysosomal enzyme with phosphotransferase. EMBO J. 16, 6684-6693 (1997).

16. Coutinho, M. F., Prata, M. J. \& Alves, S. Mannose-6-phosphate pathway: a review on its role in lysosomal function and dysfunction. Mol. Genet. Metab. 105, 542-550 (2012).

17. Padilla-Parra, S. \& Tramier, M. FRET microscopy in the living cell: different approaches, strengths and weaknesses. BioEssays 34, 369-376 (2012).
18. Topol, I., Collins, J. \& Nemukhin, A. Modeling spectral tuning in monomeric teal fluorescent protein mTFP1. Biophys. Chem. 149, 78-82 (2010).

19. Nagai, T. et al. A variant of yellow fluorescent protein with fast and efficient maturation for cell-biological applications. Nat. Biotechnol. 20, 87-90 (2002).

20. Lakadamyali, M., Rust, M. J. \& Zhuang, X. Ligands for clathrin-mediated endocytosis are differentially sorted into distinct populations of early endosomes. Cell 124, 997-1009 (2006).

21. Padilla-Parra, S., Auduge, N., Coppey-Moisan, M. \& Tramier, M. Quantitative FRET analysis by fast acquisition time domain FLIM at high spatial resolution in living cells. Biophys. J. 95, 2976-2988 (2008).

22. Yamada, H. et al. Dynamic interaction of amphiphysin with N-WASP regulates actin assembly. J. Biol. Chem. 284, 34244-34256 (2009).

23. Blanz, J. et al. Disease-causing mutations within the lysosomal integral membrane protein type 2 (LIMP-2) reveal the nature of binding to its ligand beta-glucocerebrosidase. Hum. Mol. Genet. 19, 563-572 (2010).

24. Dvir, H. et al. X-ray structure of human acid-beta-glucosidase, the defective enzyme in Gaucher disease. EMBO Rep. 4, 704-709 (2003).

25. Gaspar, P. et al. Action myoclonus renal failure syndrome: diagnostic applications of activity-based probes and lipid analysis. J. Lipid Res. 55, 138-145 (2013).

26. Alattia, J. R., Shaw, J. E., Yip, C. M. \& Prive, G. G. Molecular imaging of membrane interfaces reveals mode of beta-glucosidase activation by saposin C. Proc. Natl Acad. Sci. USA 104, 17394-17399 (2007).

27. Zachos, C., Blanz, J., Saftig, P. \& Schwake, M. A critical histidine residue within LIMP-2 mediates $\mathrm{pH}$ sensitive binding to its ligand beta-glucocerebrosidase. Traffic 13, 1113-1123 (2012).

28. Chen, P. et al. Molecular determinants of enterovirus 71 viral entry: cleft around GLN-172 on VP1 protein interacts with variable region on scavenge receptor B 2. J. Biol. Chem. 287, 6406-6420 (2012).

29. Wang, X. et al. A sensor-adaptor mechanism for enterovirus uncoating from structures of EV71. Nat. Struct. Mol. Biol. 19, 424-429 (2012).

30. Ren, J. et al. Picornavirus uncoating intermediate captured in atomic detail. Nat. Commun. 4, 1929 (2013).

31. Castonguay, A. C. et al. The glycan-binding properties of the cationindependent mannose 6-phosphate receptor are evolutionary conserved in vertebrates. Glycobiology 22, 983-996 (2012).

32. Ikushima, H. et al. Internalization of CD26 by mannose 6-phosphate/insulinlike growth factor II receptor contributes to T cell activation. Proc. Natl Acad. Sci. USA 97, 8439-8444 (2000).

33. Glickman, J. N. \& Kornfeld, S. Mannose 6-phosphate-independent targeting of lysosomal enzymes in I-cell disease B lymphoblasts. J. Cell. Biol. 123, 99-108 (1993).

34. Zhao, Y. et al. Automation of large scale transient protein expression in mammalian cells. J. Struct. Biol. 175, 209-215 (2011).

35. Chen, C. M., Krohn, J., Bhattacharya, S. \& Davies, B. A comparison of exogenous promoter activity at the ROSA26 locus using a PhiiC31 integrase mediated cassette exchange approach in mouse ES cells. PloS ONE 6, e23376 (2011).

36. Zhao, Y., Malinauskas, T., Harlos, K. \& Jones, E. Y. Structural insights into the inhibition of Wnt signaling by cancer antigen 5T4/Wnt-activated inhibitory factor 1. Structure 22, 612-620 (2014).

37. Walter, T. S. et al. A procedure for setting up high-throughput nanolitre crystallization experiments. Crystallization workflow for initial screening, automated storage, imaging and optimization. Acta. Crystallogr. D. Biol. Crystallogr. 61, 651-657 (2005).

38. Mayo, C. J. et al. Benefits of automated crystallization plate tracking, imaging, and analysis. Structure 13, 175-182 (2005).

39. Otwinowski, Z. \& Minor, W. Processing of X-ray diffraction data collected in oscillation mode. Methods Enzymol. 276, 307-326 (1997).

40. Vagin, A. \& Teplyakov, A. Molecular replacement with MOLREP. Acta Crystallogr. D Biol. Crystallogr. 66, 22-25 (2010).

41. Murshudov, G. N., Vagin, A. A. \& Dodson, E. J. Refinement of macromolecular structures by the maximum-likelihood method. Acta. Crystallogr. D Biol. Crystallogr. 53, 240-255 (1997).

42. Emsley, P., Lohkamp, B., Scott, W. G. \& Cowtan, K. Features and development of Coot. Acta. Crystallogr. D Biol. Crystallogr. 66, 486-501 (2010).

43. DeLano, W. L. PyMOL: an open-source molecular graphics tool. Ccp4 Newslett. Protein Crystallogr. 40, 11 (2002).

44. Aricescu, A. R., Lu, W. \& Jones, E. Y. A time- and cost-efficient system for high-level protein production in mammalian cells. Acta Crystallogr. D Biol. Crystallogr. 62, 1243-1250 (2006).

45. Howarth, M. et al. Monovalent, reduced-size quantum dots for imaging receptors on living cells. Nat. Methods 5, 397-399 (2008).

46. Taylor, M. J., Perrais, D. \& Merrifield, C. J. A high precision survey of the molecular dynamics of mammalian clathrin-mediated endocytosis. PLoS Biol 9 , e1000604 (2011).

47. Emiliani, V. et al. Low-intensity two-dimensional imaging of fluorescence lifetimes in living cells. Appl. Phys. Lett. 83, 2471-2473 (2003). 
48. Padilla-Parra, S. et al. Quantitative comparison of different fluorescent protein couples for fast FRET-FLIM acquisition. Biophys. J. 97, 2368-2376 (2009).

49. Ai, H. W., Henderson, J. N., Remington, S. J. \& Campbell, R. E. Directed evolution of a monomeric, bright and photostable version of Clavularia cyan fluorescent protein: structural characterization and applications in fluorescence imaging. Biochem. J. 400, 531-540 (2006).

50. Leray, A., Padilla-Parra, S., Roul, J., Heliot, L. \& Tramier, M. 827Spatiotemporal quantification of FRET in living cells by fast time-domain FLIM: a comparative study of non-fitting methods. PloS ONE 8, e69335 (2013).

\section{Acknowledgements}

We thank staff at beamline I24 of Diamond Light Source for their assistance with data collection and The Cellular Imaging Core from the Wellcome Trust Centre for Human Genetics. We also thank Thomas Walter for help with crystallization; Benjamin Davies for the phiC31 integrase expression vector; and Bass Hassan for biotinylated CI-MPR material; James Brown for CI-MPR cDNA; Jonathan Elegheert for pHL-sec-mTFP1/ mVenus constructs; Elena Seiradake for SPR advice; and Max Crispin for expert advice on glycan structure. We thank the authors of reference 9 for sharing information with us ahead of publication. Y.Z. is supported by the BioStruct-X project (283570) funded by the seventh Framework Programme (FP7) of the European Commission; J.R. by the Wellcome Trust; S.P.-P. by the Nuffield Department of Medicine Leadership Fellowship and the Wellcome Trust; and E.E.F and D.I.S. by the UK Medical Research Council. This is a contribution from the UK Instruct Centre. The Wellcome Trust Centre for Human Genetics is supported by the Wellcome Trust (grant 090532/Z/09/Z).

\section{Author contributions}

All authors designed the research and wrote the paper; Y.Z., J.R. and S.P.-P. performed the experiments, and together with E.E.F. and D.I.S analysed the results.

\section{Additional information}

Accession codes: Atomic coordinates and structure factors for space group $C 222_{1}$ and $C 2$ have been deposited with the Protein Data Bank under accession codes 4Q4B and 4Q4F, respectively.

Supplementary Information accompanies this paper at http://www.nature.com/ naturecommunications

Competing financial interests: The authors declare no competing financial interests.

Reprints and permission information is available online at http://npg.nature.com/ reprintsandpermissions/

How to cite this article: Zhao, Y. et al. Lysosome sorting of $\beta$-glucocerebrosidase by LIMP-2 is targeted by the mannose 6-phosphate receptor. Nat. Commun. 5:4321 doi: $10.1038 /$ ncomms5321 (2014)

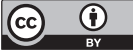

This work is licensed under a Creative Commons Attribution 4.0 International License. The images or other third party material in this article are included in the article's Creative Commons license, unless indicated otherwise in the credit line; if the material is not included under the Creative Commons license, users will need to obtain permission from the license holder to reproduce the material. To view a copy of this license, visit http://creativecommons.org/licenses/by/4.0/ 